\title{
Coherent X-rays at MAMI
}

\author{
W. Lauth ${ }^{\mathrm{a}}$, H. Backe, O. Kettig ${ }^{\mathrm{b}}$, P. Kunz, A. Sharafutdinov, and T. Weber \\ Institut für Kernphysik der Universität Mainz, D-55099 Mainz, Germany \\ / \\ Published online: 31 May 2006 - C Società Italiana di Fisica / Springer-Verlag 2006
}

\begin{abstract}
Coherent radiation in the range from soft X-rays up to hard X-rays, produced by the lowemittance electron beam of MAMI, can be used for various applications. Novel types of interferometers have been developed for the measurement of the complex index of refraction of thin self-supporting foils. For the vacuum ultraviolet and soft X-ray region the interferometer consists of two collinear undulators, and a grating spectrometer. A foil placed between the undulators causes a phase shift and an attenuation of the oscillation amplitude. The complex index of refraction has been measured at the $L_{2,3}$-absorption edges of nickel. A novel method is described for the measurement of the X-ray magnetic circular birefringence. For the hard X-ray region the interferometer consists of two foils at which the $855 \mathrm{MeV}$ electron beam produces transition radiation. Distinct interference oscillations have been observed as a function of both, the photon emission angle and the distance between the foils. The refractive index decrement $\delta(\omega)$ of a $2 \mu \mathrm{m}$ thick nickel sample foil has been measured at X-ray energies around the $K$ absorption edge at $8333 \mathrm{eV}$ and at $9930 \mathrm{eV}$ with an accuracy of better than $1.5 \%$. The line width of parametric X radiation (PXR) was measured in backward geometry with a Si single-crystal monochromator. Upper limits of the line width of $42 \mathrm{meV}, 50 \mathrm{meV}$, and $44 \mathrm{meV}$, have been determined for the (333), (444) and (555) reflections at photon energies of $5932 \mathrm{eV}, 7909 \mathrm{eV}$, and $9887 \mathrm{eV}$, respectively. Small angle scattering of the electrons in the crystal leads to a stochastic frequency modulation of the exponentially damped wave train which results in the line broadening. To elucidate the quest if the production of PXR is a kinematical or a dynamical process the radiation from silicon single-crystal targets, emitted close to the electron direction, has been studied. The observed interference structures and the narrow-band radiation in forward direction shows that PXR is produced in a dynamical process.
\end{abstract}

PACS. 07.60.Ly Interferometers - 78.20. Ci Optical constants (including refractive index, complex dielectric constant, absorption, reflection and transmission coefficients, emissivity) - 41.60.-m Radiation by moving charges $-41.50 .+\mathrm{h}$ X-ray beams and X-ray optics

\section{Introduction}

Immediately after MAMI B became fully operational for nuclear physics experiments, in the early nineties also a research program was launched to explore the potential of the high-quality, low-emittance electron beam for applications. It was our conviction that it ought to be possible to use X-rays produced with the $855 \mathrm{MeV}$ beam of MAMI in various fields of physics, material science, medicine and biology. Of course, attention focused at that time on the third-generation synchrotron radiation sources like ESRF, APS and Spring8 which were on the horizon and, in particular, on the production of brilliant soft X-ray flashes in a single pass of high-current electron bunches through an undulator by the process of self-amplified spontaneous emission (SASE). However, various other processes were

\footnotetext{
${ }^{a}$ e-mail: lauth@kph.uni-mainz.de

b Present address: Arcor AG \& Co. KG, 65760 Eschborn, Germany.
}

also considered at that time to be of interest for the production of soft and hard X-ray beams with external high-quality electron beams. The most important ones are schematically depicted in fig. 1 . These are transition radiation (TR), channeling radiation (CR), parametric X-radiation (PXR), undulator radiation (UR), and SmithPurcell radiation (SPR). There are potential advantages of such X-ray sources over synchrotron radiation sources or free electron lasers on the basis of SASE. First of all, since accelerators may become relatively inexpensive in the future, they could meet the radiation requirements of research laboratories or hospitals on the spot. Secondly, the X-ray beam can be triggered and its time structure adapted to nearly any experimental requirement. In particular, the electron beam can easily be turned off if the $\mathrm{X}$-ray beam is not used minimizing power consumption and radiation production in the beam dump.

At MAMI with UR and TR brilliant photon beams can be produced with energies covering the range between 


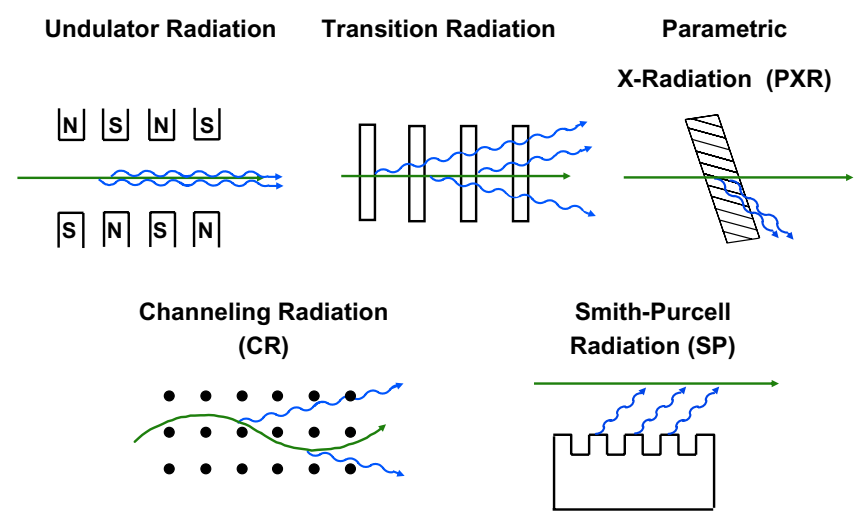

Fig. 1. Processes for the generation of coherent radiation with relativistic electrons.

some $100 \mathrm{eV}$ and up to about $50 \mathrm{keV}$. Brilliance means that a large number of photons from a small source spot size down to the $\mu \mathrm{m}$ range are emitted with high directionality in space. In particular, the hard TR X-ray beam turned out to be comparable in photon flux and brilliance with second-generation synchrotron radiation sources [1]. Taking advantage of this fact a novel $K$-edge imaging system [2] was developed. In addition, X-ray phase contrast imaging has been accomplished. For the latter we refer to the contribution ref. [3] in this issue.

SPR is generated when a beam of charged particles passes close to the surface of a periodic structure. This type of radiation has been investigated in the optical spectral range with the $855 \mathrm{MeV}$ beam of MAMI [4]. A detailed discussion of the emitted photon number per electron led to the conclusion that a SPR source is not advantageous in comparison with an UR source for ultrarelativistic beam energies. At present experiments are being performed at the $3.4 \mathrm{MeV}$ injector LINAC of MAMI to explore the generation of intense SPR in the $\mathrm{THz}$ region of the electromagnetic spectrum [5].

In channeling, the charged particle directions are closely aligned with an atomic row or with crystal planes, and their motion is governed by many correlated collisions with crystal atoms. As a result, the particles are steered along strings or planes and CR is emitted. Channeling experiments have been taken up at MAMI only very recently, see ref. [6]. They are connected with the feasibility of a crystalline undulator with positrons which was investigated recently in great detail [7].

In sect. 2 of this contribution our results obtained with a novel interferometry principle will be reviewed. The interferometer consists of two spatially separated, phasecorrelated radiation sources in the soft and hard X-ray ranges. For soft X-rays the radiation sources are undulators with small period length, for hard X-rays they are foils in which the electron beam produces TR. It will be shown in this section 2 that the optical properties of foils can be determined in the soft and hard X-ray region.

If the electron beam strikes a crystal, it emits quasimonochromatic PXR close to a Bragg angle. This kind of radiation source is amazing for its compactness, since production and monochromatisation of the radiation take place in the same crystal. The expected small spectral line width of PXR would promise an abundance of application possibilities, e.g. within the field of solid-state physics. However, line broadening by multiple scattering of the electrons in the crystal may spoil the superb line width. In sect. 3 our experiments addressing this question are reviewed, including fundamental aspects like the question whether the process of PXR production is of kinematical or of dynamical nature.

The paper closes with a conclusion and an outlook.

\section{X-Ray interferometry}

Resonant anomalous X-ray scattering plays an increasingly important role in many disciplines of physics, biology, and material sciences. Using the brilliant and tuneable X-ray beams from modern synchrotron radiation sources, it is now possible to fully exploit the information in the strong energy and polarisation dependence of the atomic scattering amplitude $f(\omega, \boldsymbol{q})=f_{0}(\boldsymbol{q})+$ $f^{\prime}(\omega)+i f^{\prime \prime}(\omega)$ near absorption edges [8,9]. This microscopic description can be translated into a macroscopic description with the complex index of refraction $n(\omega)=$ $1-\delta(\omega)+i \beta(\omega)$ by the relations for the refractive index decrement $\delta(\omega)=(1 / 2)\left(\omega_{p} / \omega\right)^{2}\left(f_{0}(0)+f^{\prime}(\omega)\right) / Z$ and the absorption index $\beta(\omega)=(1 / 2)\left(\omega_{p} / \omega\right)^{2} f^{\prime \prime}(\omega) / Z$. In these expressions $Z$ is the atomic number, $\omega_{p}$ the plasma frequency with $\omega_{p}^{2}=4 \pi r_{0} c^{2} n_{a} Z, r_{0}$ the classical electron radius, $n_{a}$ the number of atoms per volume, and $f_{0}(0)=Z$ neglecting relativistic corrections.

The imaginary part of the scattering amplitude $f^{\prime \prime}$ can be directly determined from the total photon cross section $\sigma(\omega)$ by employing the optical theorem: $f^{\prime \prime}(\omega)=$ $\omega \sigma(\omega) /\left(4 \pi r_{0} c\right)$. The total cross section is well approximated by the absorption cross section which can be measured by a transmission experiment. The real part $f^{\prime}$ can be calculated from $f^{\prime \prime}$ by means of Kramers-Kronig dispersion relations. However, this method is suited for a relative comparison only, since it requires precise absorption data for all frequencies from zero to infinity [10]. If precise absolute values are needed, a direct measurement of $f^{\prime}(\omega)$ is required. Direct measurements are based on X-ray interferometry with the Bonse Hard-X-ray interferometer [11], refraction through a prism $[12,13]$, diffraction from perfect crystals and pendellösung fringes [14,15], determination of the angle of total reflection $[10,16]$, and Fresnel bi-mirror interferometry [17]. Whereas most of these methods are based on splitting of either wave amplitudes or wave fronts the novel type of interferometer which is described here uses two spatially separated coherent X-ray emitters.

The basic idea of the interferometer will be explained by means of the schematic experimental setup shown in fig. 2. Relativistic electrons create two wave trains in source 1 and source 2 , the relative distance $\Delta$ of which is given in leading order by $\Delta(\theta, d)=\frac{1}{2}\left(\gamma^{-2}+\theta^{2}\right) d$. Here $d$ is the distance between the sources, $\gamma$ the Lorentz factor of the electron, and $\theta$ the observation angle with respect to the electron beam direction. The monochromator serves 


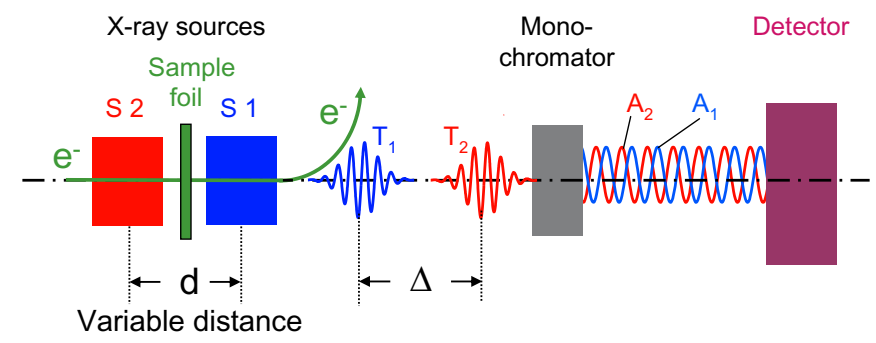

Fig. 2. Interferometry with spatially separated coherent X-ray emitters.

as a Fourier analyser of the wave trains. The two resulting plane waves have a phase difference of $\Phi=\frac{\omega}{v} \Delta(\theta, d)$ ( $v$ is the velocity of the electron) and interfere in the detector, resulting in oscillations of the intensity $I(d)$, if the distance $d$ is varied. A sample foil placed between the two sources produces an additional phase shift and attenuation of wave 2. Consequently, both quantities, i.e. the refractive index decrement $\delta$ and the absorption index $\beta$, can be extracted from the measured interference oscillations $I(d)$ with and without the foil between the sources. This holds independently of the nature of the emission process, provided that the produced X-rays remain coherent.

\subsection{The soft $X$-ray interferometer}

For photon energies in the range of about $100 \mathrm{eV}$ up to $2 \mathrm{keV}$ we use two identical undulators (period length $L_{U}=$ $12 \mathrm{~mm}$, number of periods 10 , undulator parameter $K=$ 1.1) and a grating spectrometer. The recorded intensity with a foil between the undulators is given by

$$
\begin{aligned}
I(d)= & \left|A_{1}\right|^{2}+\left|A_{2}\right|^{2} e^{-2 \frac{\omega}{c} \beta(\omega) t_{0}}+2\left|A_{1}\right|\left|A_{2}\right| e^{-\frac{\omega}{c} \beta(\omega) t_{0}} \\
& \times \cos \left\{\frac{\omega}{c}\left[\Delta(\theta, d)+\delta(\omega) t_{0}+\frac{K^{2}}{4 \gamma^{2}} L_{U}\right]\right\}
\end{aligned}
$$

with $A_{2}$ being the amplitude of the upstream undulator, $A_{1}$ that of the downstream one and $t_{0}$ the thickness of the foil.

The interferometer has been developed at the Mainz Microtron MAMI and its performance was demonstrated with measurements at the $K$ absorption edge of carbon at $284 \mathrm{eV}$. Details of this experiment can be found elsewhere [18]. The visibility (coherence), defined by $C=$ $\left(I_{\max }-I_{\min }\right) /\left(I_{\max }+I_{\min }\right)$ without sample foil, is close to its maximum value $C=1$. No loss of coherence was observed over the scanning distance of $15 \mathrm{~cm}$. Therefore, the optical constants $\delta$ and $\beta$ could be extracted by a fit with simple cosine functions.

Measurements were also performed at the $L_{2}$ absorption and $L_{3}$-absorption edges of nickel at $871 \mathrm{eV}$ and $855 \mathrm{eV}$, respectively. The experimental setup was similar with that described in ref. [18] with the following modifications: the third harmonics of the undulator was chosen as radiation, which was generated at an electron energy of $766.3 \mathrm{MeV}$. The radiation was analyzed with a variable line spacing (VLS) grating [19] with an energy

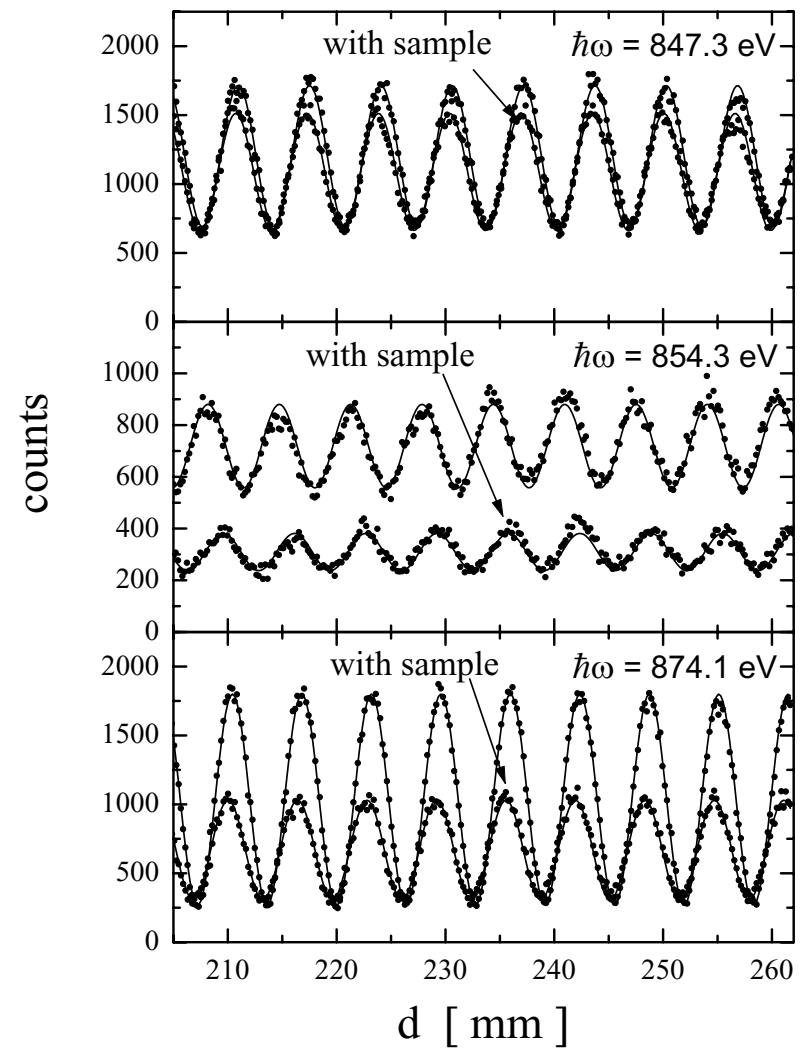

Fig. 3. Intensity oscillations as a function of the distance $d$ between the undulators with and without the self-supporting $83.2 \mu \mathrm{g} / \mathrm{cm}^{2}$ nickel sample foil at three different photon energies at the $L_{2,3}$ absorption edges. Note the change of sign of the phase shift at the photon energy of $854.3 \mathrm{eV}$.

resolution of $0.44 \mathrm{eV}$. Typical measured intensity oscillations are shown in fig. 3. The extracted optical constants at the $\mathrm{Ni} L_{2,3}$ are shown in fig. 4 . A high accuracy has been reached, even in the region where $\beta \geq \delta$ in which the optical constants can be determined from reflectivity measurements only with large uncertainties [20]. The remarkable fact about this measurement is the hight of the $L_{3}$ resonance with its maximum value $\beta=(5.64 \pm 0.24) \cdot 10^{-3}$. This value corresponds to an imaginary scattering factor $f^{\prime \prime}=65.7 \pm 2.8$. In ref. [21] a mass attenuation coefficient $\mu=2 \cdot 10^{4} \mathrm{~cm}^{2} / \mathrm{g}$ was determined from which, with the relation $\mu=(4 \pi) \cdot \beta$, an $f^{\prime \prime}=24$ can be calculated with an estimated uncertainty of $10 \%$. The difference may originate from the better resolution in our experiment. Correlated with this $L_{3}$ absorption resonance is a change of sign of the refractive index decrement $\delta(\omega)$. It is interesting to notice that for $\delta(\omega)<0$ the real part of the refractive index $1-\delta(\omega)$ is larger than 1 and a monochromatic Cherenkov radiation with an energy of $855 \mathrm{eV}$ will be emitted. This fact has been pointed out in the literature.

The strong absorbtion line at the $L_{3}$ edge of $\mathrm{Ni}$ is the result of an allowed dipole transition between the $2 p_{3 / 2}$ core state and empty $3 \mathrm{~d}$ valence states above the Fermi energy. This transition exhibits a strong X-ray Magnetic Circular Dichroisim (XMCD) effect which can 


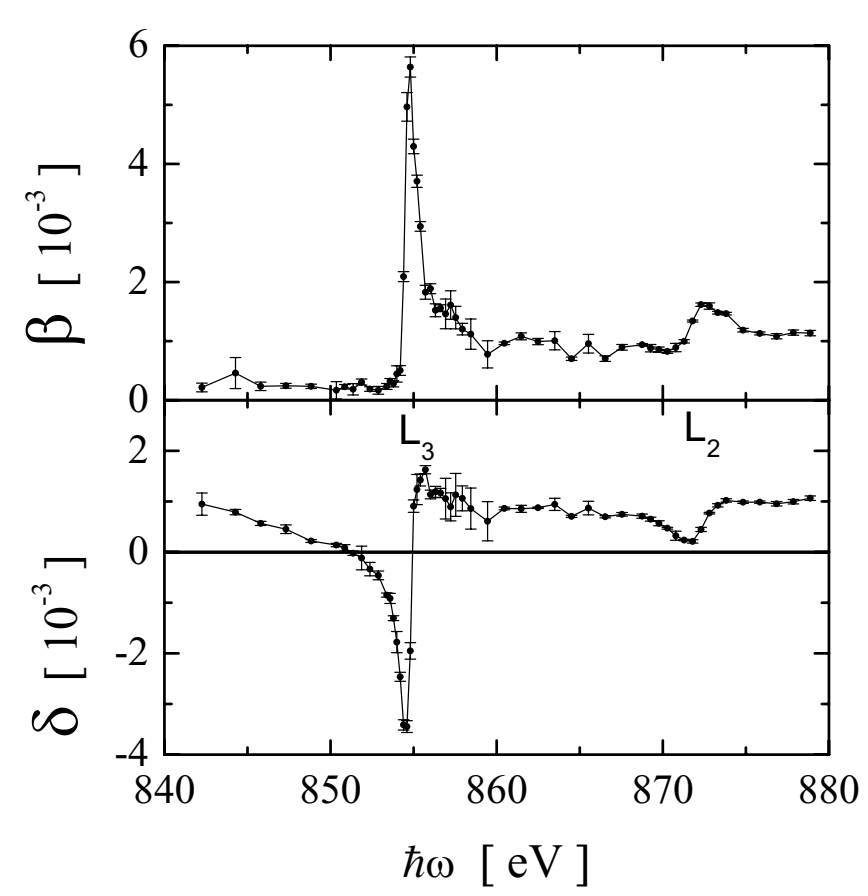

Fig. 4. Absorption index $\beta$ and refraction index decrement $\delta$ of an $83 \mu \mathrm{g} / \mathrm{cm}^{2} \mathrm{Ni}$ sample foil as obtained from the analysis of the intensity oscillation measurements as shown in fig. 3 . The photon energy resolution was $0.44 \mathrm{eV}$.

be used to probe the magnetic properties of the material [22]. With XMCD spectroscopy, pioniered by Schütz and coworkers [23], the difference in the absorption of left and right circular polarized light is measured. Connected with the XMCD is the X-ray Magnetic Circular Birefringence (XMCB) also known as Faraday Magneto-Optical Rotation (MOR). Both effects can be described with the complex index of refraction $n_{ \pm}(\omega)=1-\delta_{ \pm}(\omega)+i \beta_{ \pm}(\omega)$ for the two \pm helicity states of the radiation. Thickness variation in the transmission measurements hampered the accuracy in the determination of $\beta_{ \pm}(\omega)$ with the XMCD spectroscopy, especially near the strong-absorption lines where the XMCD effect is the largest. It has been shown in ref. [24], that such thickness effects are less important for the measurement of the refractive index $\delta_{ \pm}(\omega)$. However, this measurement is difficult because of the lack of polarization analyzers in the soft X-ray region.

Our interferometer has been developed further to measure $\delta_{ \pm}(\omega)$ without any polarization filter. The magnetized sample foil with the magnetization direction parallel or antiparallel to the electron beam axis was positioned between the two undulators, see fig. 5. Due to the XMCB and XMCD effect, the linear polarized light from the first undulator suffers a helicity-dependent phase shift and absorption resulting in elliptical polarized light. The major semi-axis of the ellipse is rotated by an angle $\psi$ with respect to the plane in which the impinging linear polarized $\boldsymbol{E}$ field vector oscillates. The second undulator which can be rotated around the electron beam axis acts as the analyzer. The maximum visibility determines the rotation angle $\psi$ of the ellipse on which the

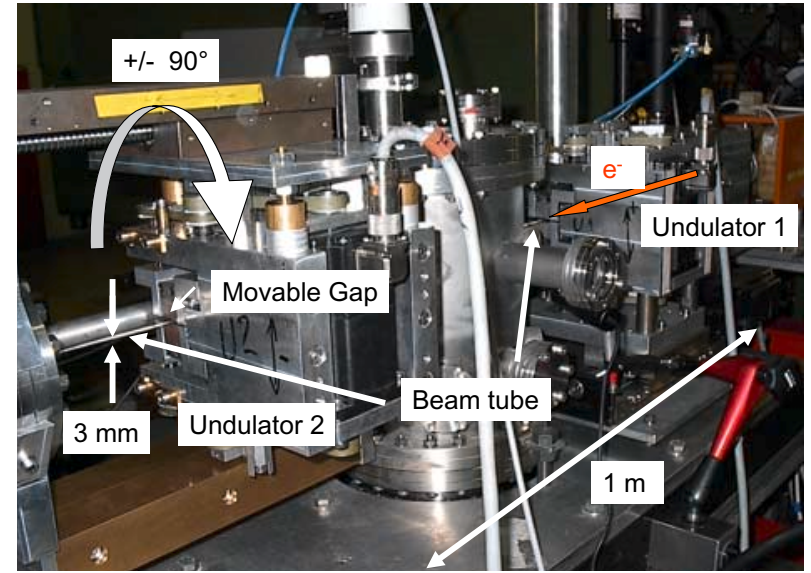

Fig. 5. Experimental setup of the new undulator interferometer. The undulator 2 can be both moved along and rotated around the electron beam axis. These possibilities allow production of radiation with a well-defined polarization state between linear and circular. In addition, the undulator gap can be changed for an online variation of the photon energy via the undulator parameter $K$. Note that the electron beam traverses a vacuum tube of only $3 \mathrm{~mm}$ in diameter over a length of about $1 \mathrm{~m}$.

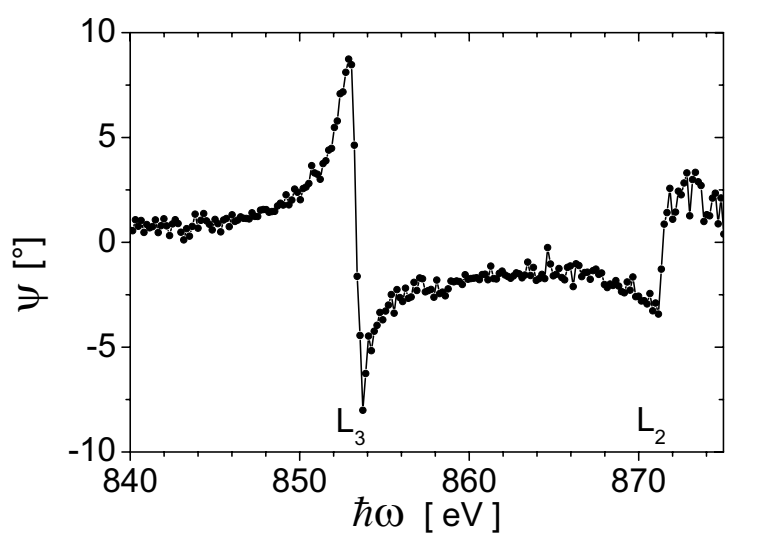

Fig. 6. Preliminary results of a measurement of the rotation angle $\psi$ of the ellipse on which the $\boldsymbol{E}$ field vector rotates behind a $72.5 \mathrm{~nm}$ self-supporting $\mathrm{Ni}$ foil, which was illuminated with a linear polarized undulator radiation. Shown are measurements at the $L_{3}$ and $L_{2}$ absorption edges.

$\boldsymbol{E}$ field vector moves behind the foil. This angle is given by $\psi=\frac{\omega}{2 c} t_{0}\left(\delta_{+}(\omega)-\delta_{-}(\omega)\right)$. The first measurements of the angle $\psi$ of a $72.5 \mathrm{~nm}$ magnetized $\mathrm{Ni}$ foil, placed in a magnetic field of $1.0 \mathrm{~T}$, are shown in fig. 6. In this experiment the photons were detected in the energy dispersive plane of the grating spectrometer with a windowless CCD-detector [25]. The visibility for rotation angles between $-105^{\circ}$ and $+105^{\circ}$ of the second undulator was extracted from the interference oscillation as a function of the distance between the undulators. Furthermore, also the refractive index decrement $\beta_{ \pm}(\omega)$ can be extracted simultaneously from this measurement which allows the full determination of the helicity-dependent complex index of 


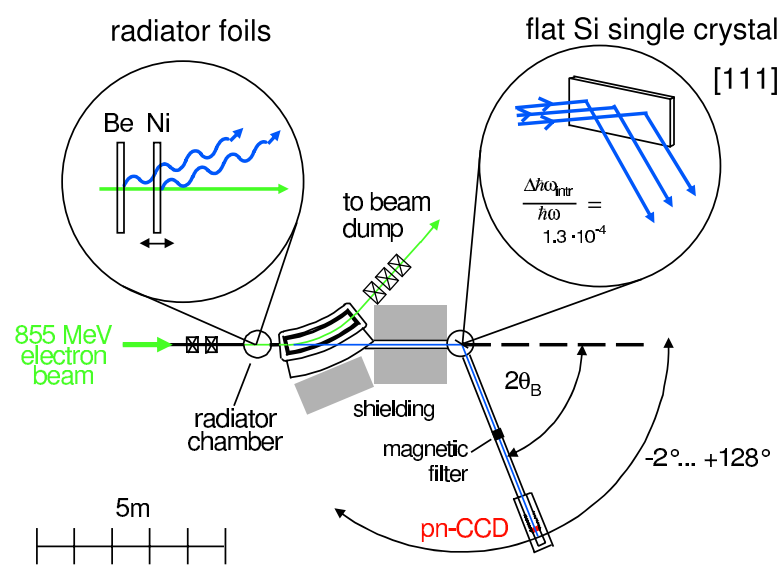

Fig. 7. Experimental setup of the transition radiation interferometer. The monochromator is a flat silicon single crystal, cut with the (111) plane parallel to the surface, at a distance of $5.5 \mathrm{~m}$ from the foils. It acts as energy dispersive mirror. A silicon $1 \times 3 \mathrm{~cm}^{2}$ pn CCD with active thickness of $300 \mu \mathrm{m}$ and a pixel size of $150 \times 150 \mu \mathrm{m}^{2}$ was used as detector [26]. The CCD is located at a distance of $5.5 \mathrm{~m}$ from the monochromator crystal.

refraction $n_{ \pm}(\omega)$ without knowledge of the degree of the polarization of the light.

\subsection{The hard X-ray interferometer}

When a charged particle traverses the interface of two media with different dielectric polarizability, transition radiation (TR) is emitted. The TR radiation is sharply peaked into forward direction with a characteristic opening angle of about $2 / \gamma$ and features broadband characteristics with a cut-off energy at about $40 \mathrm{keV}$. It is well known that the TR amplitudes from two interfaces of a single foil interfere coherently. The same holds for the amplitudes from two or more foils (interfoil interference), see, e.g., ref. [27]. It was suggested more than fifteen years ago by Moran et al. [28] that the interfoil coherence of TR, generated by relativistic electrons, constitutes a new technique for the measurement of the refractive index decrement $\delta(\omega)$ in the X-ray region. A number of experiments were performed in the soft and hard X-ray region in which more or less clear interference structures were observed [28,29, $30,31,32,1]$. However, that $\delta(\omega)$ really can be measured by such a technique has been demonstrated first by the X-ray interferometer described in this work. It consists of two foils at which the $855 \mathrm{MeV}$ MAMI beam produces transition radiation, a single crystal spectrometer with a flat crystal in Bragg geometry, and a pn CCD X-ray detector, see fig. 7. Details of the experiment have been reported elsewhere $[33,34,35]$.

Measurements have been performed on nickel around the $K$ absorption edge at $8333 \mathrm{eV}$ as well as around $9930 \mathrm{eV}$, well above the $K$ absorption edge, where extended diffraction anomalous fine structures (EDAFS) in the dispersion spectra are supposed to be negligible. A

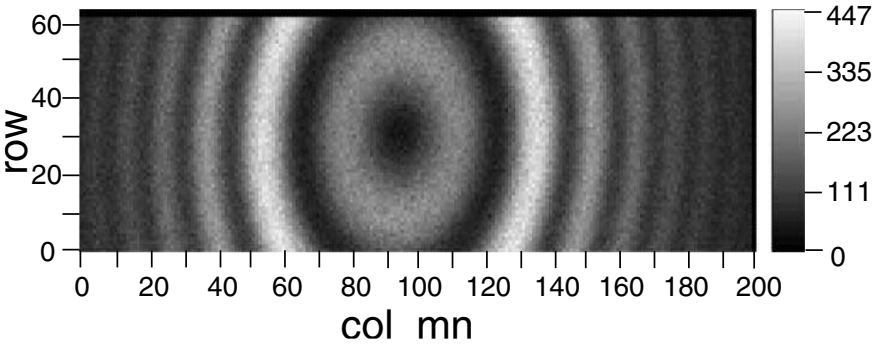

Fig. 8. Measured interference pattern at a fixed foil distance $d=475.0(2) \mu \mathrm{m}$. The central photon energy $\hbar \omega=9929 \mathrm{eV}$ is well above the $K$ absorption edge of nickel. Grey levels indicate the intensities.

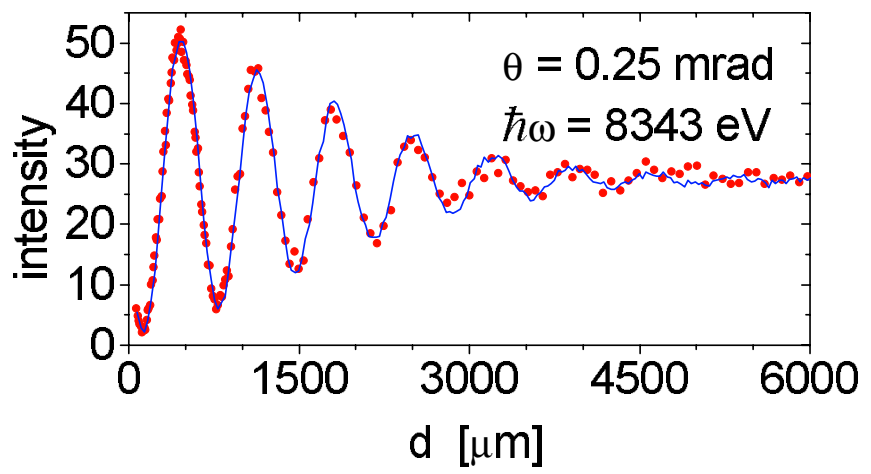

Fig. 9. Examples of interference oscillations as a function of foil distance $d$ at a fixed photon energy $\hbar \omega$ and a fixed observation angle $\theta$ as indicated. The ordinates are counts $/\left(\mathrm{ms} \mathrm{mrad}^{2}\right)$. The measurements (dots) are well met by the simulation calculation (full line).

typical example of a measured TR interference pattern is shown in fig. 8.

There are two possibilities to extract $\delta_{1}$ of the downstream nickel foil from the TR interference patterns shown in fig. 8. In the first one the information is obtained from the interference oscillations as a function of the observation angle $\theta$ at fixed distance between the foils. Since the pn CCD detector was arranged horizontally the interference oscillations were observed essentially along the energy dispersive angular coordinate $\theta_{x}$. Reliable results can be obtained if $\delta_{1}(\omega)$ can be approximated with reasonable accuracy by a linear expansion as a function of the photon energy $\hbar \omega$. Close to an absorption edge such an approximation is not valid. In such a case, as a second possibility, $\delta_{1}(\omega)$ can be extracted from the interference oscillations observed as a function of foil distance $d$, as shown in fig. 9 . The rapid damping of the visibility after about three periods originates mainly from the small-angle scattering of the electrons in the upstream foil which was made of the low- $Z$ element beryllium to minimize the effect. The results of the refractive index decrement $\delta_{1}(\omega)$ around the $K$ absorption edge of $\mathrm{Ni}$ are shown in fig. 10. Details of the analysis procedure can be found in refs. [34,36].

The measured $\delta_{1}(\omega)$ agrees at the $K$ absorption edge within the total error of $\Delta \delta_{1} / \delta_{1} \leq 1.5 \%$, with the Kramers-Kronig transformation of $\beta_{1}(\omega)$ of refs. [37,38]. 


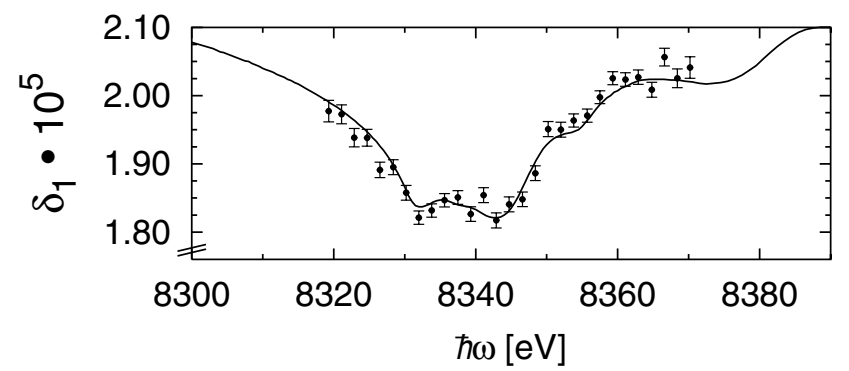

Fig. 10. Refractive index decrement $\delta_{1}(\hbar \omega)$ at the $K$ absorption edge as obtained from interference oscillations. The error bars represent pure statistical errors of the experiment. The full line is a Kramers-Kronig transform of the absorption data [37, $38]$.

In comparison, the measurements of $\delta_{1}(\omega)$ by Bonse and co-workers $[39,40]$ with Bonse-Hart interferometers [11] are systematically too low by about $1.5 \%$. This small deviation may originate from a systematical error in the foil thickness measurements in those experiments.

\section{Parametric X-radiation}

Parametric X-radiation (PXR) or quasi-Cherenkov radiation is produced when a relativistic electron traverses a single crystal, and the wave vector $\boldsymbol{k}_{v}$ of the virtual photon associated with the electron field, the reciprocal lattice vector $\boldsymbol{H}$ of a specific crystal plane and the wave vector $\boldsymbol{k}_{r}$ of the emitted X-ray nearly fulfil the well-known diffraction condition $\boldsymbol{k}_{v}+\boldsymbol{H} \cong \boldsymbol{k}_{r}$. For ultra-relativistic electrons the wave vector $\boldsymbol{k}_{v}$ nearly coincides with the electron velocity vector $\boldsymbol{v}$ and the emission process can also be understood as diffraction of virtual photons by the crystal. The production mechanism of PXR in such a medium with three-dimensional periodic permittivity was extensively studied both theoretically by Baryshevsky and Feranchuk [41,42], Garibyan and Yang [43,44], and TerMikaelian [45] and experimentally by a large number of researchers. For an overview of the theoretical and experimental work up to the year 1997 see, e.g., ref. [46] and also ref. $[47,48,49,50]$ for the recent works.

PXR features a sharp quasi-monochromatic X-ray beam close to the Bragg angle with a very narrow line width. The angular distribution consists of one peak above and one below the symmetry plane of the crystal. Their spatial widths are characterized by the angle $\theta_{p h}=\left(1 / \gamma^{2}+\left(\omega_{p} / \omega\right)^{2}\right)^{1 / 2}$, with $\gamma$ the Lorentz factor of the moving particle, $\omega$ the angular frequency of the emitted photon, and $\omega_{p}$ the plasma frequency of the crystal, with $\hbar \omega=31 \mathrm{eV}$ for Si. The theoretical description of the intensity distribution of PXR [51,52,45,53,54], suitably modified for self-absorption and multiple-scattering effects, has been tested for a broad range of electron energies extending from $3.5 \mathrm{MeV}[55,56]$ to about $1 \mathrm{GeV}[46]$. It was found to be accurate within $12 \%$.

The line width $W_{\text {nat }}$ of PXR is in essence determined by the photo absorption in the crystal, if the electron beam divergence, the small-angle electron scattering in the crystal and the solid angle of the X-ray detector can all be neglected, and the crystal has a perfect lattice structure. An exponentially damped wave train is emitted of which the Fourier transform is a Lorentzian with width [54]

$$
W_{n a t}=-\frac{\chi_{0}^{\prime \prime}}{2 \sin ^{2} \theta_{0}} \cdot \hbar \omega_{0} .
$$

The quantity $\chi_{0}^{\prime \prime}$ is the imaginary part of the mean dielectric susceptibility $\chi_{0}=\chi_{0}^{\prime}+i \chi_{0}^{\prime \prime}$. This line width $W_{\text {nat }}$ is called "natural line width". For example, the (444) reflection of silicon at $\hbar \omega_{0}=7908 \mathrm{eV}$ yields $\chi_{0}^{\prime \prime}=-3.74$. $10^{-7}$ [57]. With this value a natural line width $W_{\text {nat }}=$ $1.48 \mathrm{meV}$ results from eq. (2) for backward emission, i.e. for $\theta_{0} \simeq \pi / 2$. It is interesting to note that the corresponding Darwin-Prins curve has a width of $W_{D P}=38.5 \mathrm{meV}$ and is a factor of 26 broader, see also fig. 13. In view of the fact that such a narrow bandwidth source could be of extreme interest for many applications, a number of experiments were performed to determine the line width of PXR. Measurements at the low electron beam energy of $6.8 \mathrm{MeV}$ result in a line width of $48 \mathrm{eV}$ for a $55 \mu \mathrm{m}$ thick diamond crystal at a photon energy of $8.98 \mathrm{keV}$ [58]. This rather large line width originates from the multiple scattering of electrons in the crystal. With the critical absorber technique experiments have been performed at MAMI at an electron beam energy of $855 \mathrm{MeV}$ [59]. Upper limits of the line width of $1.2 \mathrm{eV}$ and $3.5 \mathrm{eV}$ have been determined for the (111) and (022) reflections of silicon single crystals at photon energies of $4966 \mathrm{eV}$ and $8332 \mathrm{eV}$. These limits originate mainly from geometrical line broadening effects.

In backward geometry geometrical line broadening contributions, originating from the angular spread of the electron beam and small-angle scattering of the electrons in the crystal, minimize. To investigate whether under these conditions line widths as small as the Darwin-Prins values can be reached, experiments have been performed at MAMI which will be described in the next subsection.

\subsection{Measurements of the line width of PXR}

Measurements of the line shape of PXR were done with the $855 \mathrm{MeV}$ electron beam of MAMI [60]. The experimental setup is shown in fig. 11 . The backward-emitted radiation of a reflection from an (nnn) plane was analyzed with a silicon single-crystal monochromator in Bragg geometry at the same (nnn) reflection. A vertical slit of $2 \mathrm{~mm}$ width in front of the analyzer crystal reduces the divergence of the $\mathrm{X}$-rays to $0.07 \mathrm{mrad}$. The $\mathrm{PXR}$ radiation was separated from the backward-diffracted transition radiation (DTR) by tilting the target crystal to $\psi_{x} / 2=5 \mathrm{mrad}$ for which PXR emission dominates in comparison to DTR.

The measured line shapes are shown in fig. 12 for various reflections. The lines are convolutions of the PXR emission spectra, which are broadened by multiple scattering of the electrons, and the response function of the analyzer crystal. The data were analyzed assuming a Gaussian distribution for the broadened PXR line and the DarwinPrins curve of the analyzer crystal. The best fits are shown 


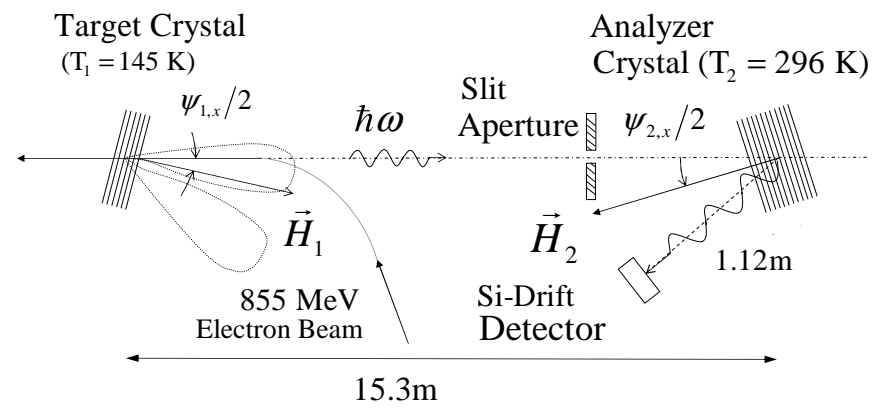

Fig. 11. Experimental setup. The target crystal was cooled down to a temperature of $T_{1}=145 \mathrm{~K}$, while the analyzer crystal was kept at room temperature $T_{2}=296 \mathrm{~K}$. Since the lattice parameter $a(T)$ of the crystal diminishes by cooling, an enlarged photon energy and an enlarged deflection angle $\psi_{2, x}=45.6 \mathrm{mrad}$ at $\psi_{1, x}=0$ result. The angle is large enough for a lateral displacement of the Si-drift detector that it does not shadow the radiation to be analyzed. A $1 \mathrm{~mm}$ thick silicon single crystal with an area of $30 \mathrm{~mm} \times 20 \mathrm{~mm}$, cut with the (111) plane parallel to the surface, was used as analyzer crystal.

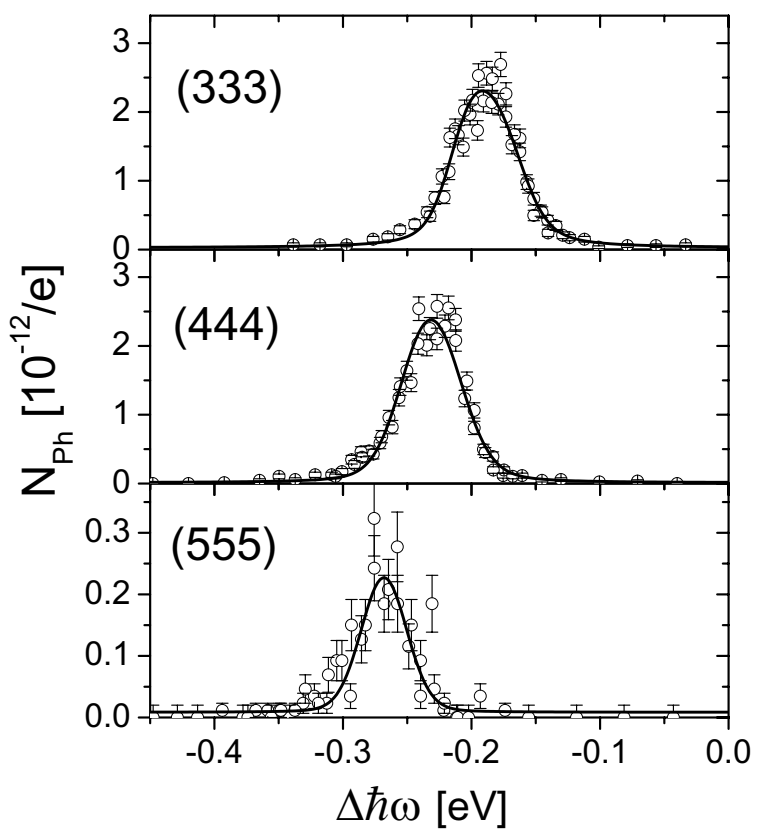

Fig. 12. Line shape measurements of PXR at $\psi_{1, x} / 2=5 \mathrm{mrad}$ for different reflections as indicated by (nnn) values; $\Delta \hbar \omega=$ $\hbar \omega-\hbar \omega_{0}$, with $\hbar \omega_{0}$ the energy of the DTR reflection.

in fig. 12 by the full lines. The resulting PXR widths are shown in fig. 13 together with the natural line width according to eq. (2) and the width of the Darwin-Prins curve. Only for the (333) reflex the observed line width is smaller than the width of the Darwin-Prins curve. It could not be excluded that imperfections of the analyzer crystal itself broaden the higher reflections. Therefore, the real PXR line could be somewhat smaller as the fit results shown in fig. 13.

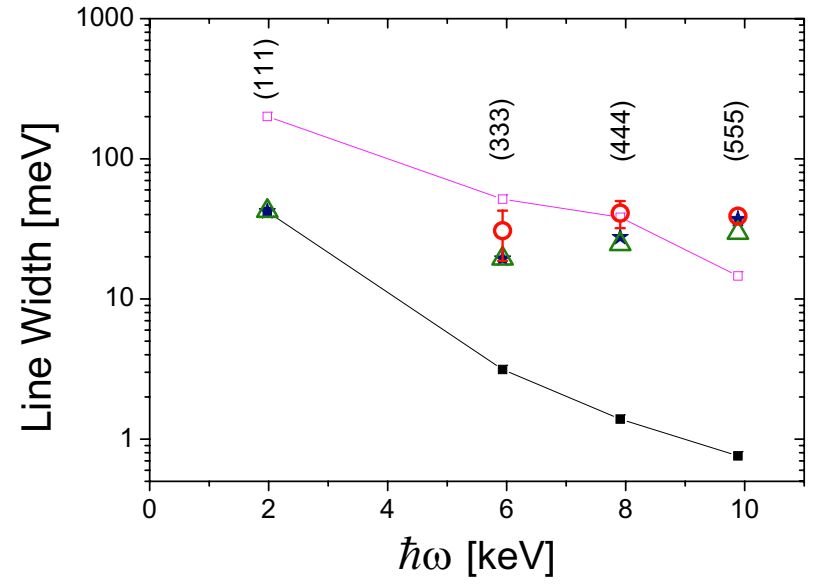

Fig. 13. Measured and calculated line widths (FWHM) for various (nnn) reflections for a silicon single crystal of $525 \mu \mathrm{m}$ thickness. Open circles: measured PXR line width, full squares: natural PXR line widths for straight electron trajectories, open squares: Darwin-Prins width, triangles: line widths calculated with the analytical model of ref. [48], and stars: Monte Carlo simulations.

It has been discussed in ref. [48] that the small-angle scattering of the electron in the Coulomb potential of the crystal atoms results in a stochastic change of the electron direction which leads to a stochastic frequency modulation of the exponentially damped wave train of PXR. As a consequence, the PXR line broadens. The scattering distribution function was approximated by a Gaussian. This approximation may not be anymore valid if the electron enters the crystal close to a channeling axis or a channeling plane. In such cases Monte Carlo simulations of the scattering process must be applied to obtain the PXR wave train. However, it is interesting to notice that calculations according to ref. [48] as well as also Monte Carlo simulated line shapes, which are both shown also in fig. 13, are in good agrement with the measurements.

\subsection{Measurement of forward-diffracted PXR}

Up to now it could not be decided experimentally whether PXR emission is a kinematical or a dynamical process. The reason has been discussed by Nitta in a recent paper [61]. He showed that the first-order approximation of the dynamical calculation gives the kinematical expression. Extremely accurate absolute intensity measurements would be required to figure out a difference. Baryshevsky [62] proposed to search for the predicted forwarddiffracted wave (FDPXR) which is associated to PXR and emitted close to the direction of the electron propagation. Similar proposals have also been communicated by Nasonov $[63,64]$. In ref. [65] the observation of narrow FDPXR structures from a $410 \mu \mathrm{m}$ thick tungsten single crystal at photon energies of 28.3 and $40 \mathrm{keV}$ is reported.

At the Mainz Microtron MAMI experiments were performed for the search of the forward-diffracted wave (FDPXR) in single silicon crystals of various thicknesses [66]. 


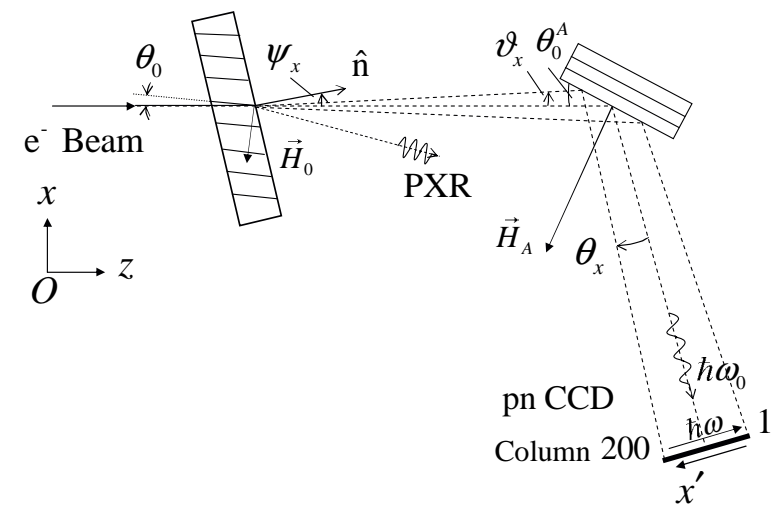

Fig. 14. Schematic experimental setup for the search of FDPXR.
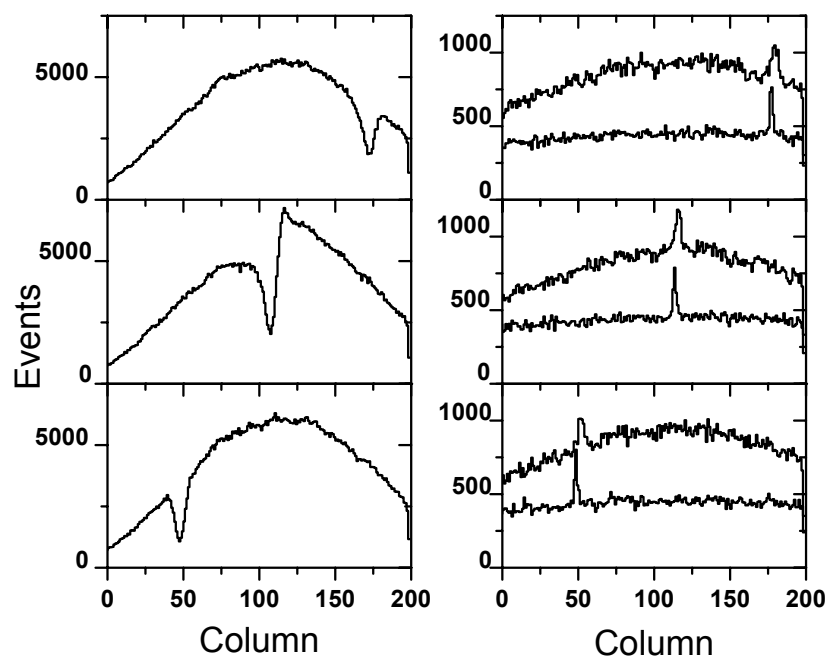

Fig. 15. Measurements at photon energy of $10.554 \mathrm{keV}$ and target thickness of $58 \mu \mathrm{m}$ (left) and $1000 \mu \mathrm{m}$ (right). Shown are intensity distributions summed over all 64 rows of the pn CCD detector as a function of the column number. From the upper to the lower panel the rotation angle $\psi_{x}$ of the target crystal was varied in steps $\delta \psi_{x}=0.5894 \mathrm{mrad}$. Beam current: $53.5 \mathrm{nA}$, exposure time: $600 \mathrm{~s}$. Left panel: Beam spot size about $500 \mu \mathrm{m}$ (FWHM) horizontally and $434 \mu \mathrm{m}$ (FWHM) vertically. The destructive interference fringes can clearly be recognized. Right panel, upper curves: Beam spot size about $500 \mu \mathrm{m}$ (FWHM) horizontally and $434 \mu \mathrm{m}$ (FWHM) vertically. Right panel, lower curves: Reduced beam spot size $114 \mu \mathrm{m}$ (FWHM) horizontally and $200 \mu \mathrm{m}$ (FWHM) vertically.

The basic idea of the experiment will be explained by means of fig. 14. A silicon single-crystal target was positioned in such a way that the PXR reflex at a photon energy $\hbar \omega_{0}=10.554 \mathrm{keV}$ is located at twice the Bragg angle $\theta_{0}=10.797^{\circ}$ in the horizontal plane of drawing. The radiation in forward direction close to the electron direction was analyzed with a flat silicon single-crystal monochromator in combination with a pn CCD camera as a position-sensitive and energy-resolving photon detector. The quasi-monochromatic FDPXR peak energy matches with the energy of the analyzer crystal at only one specific
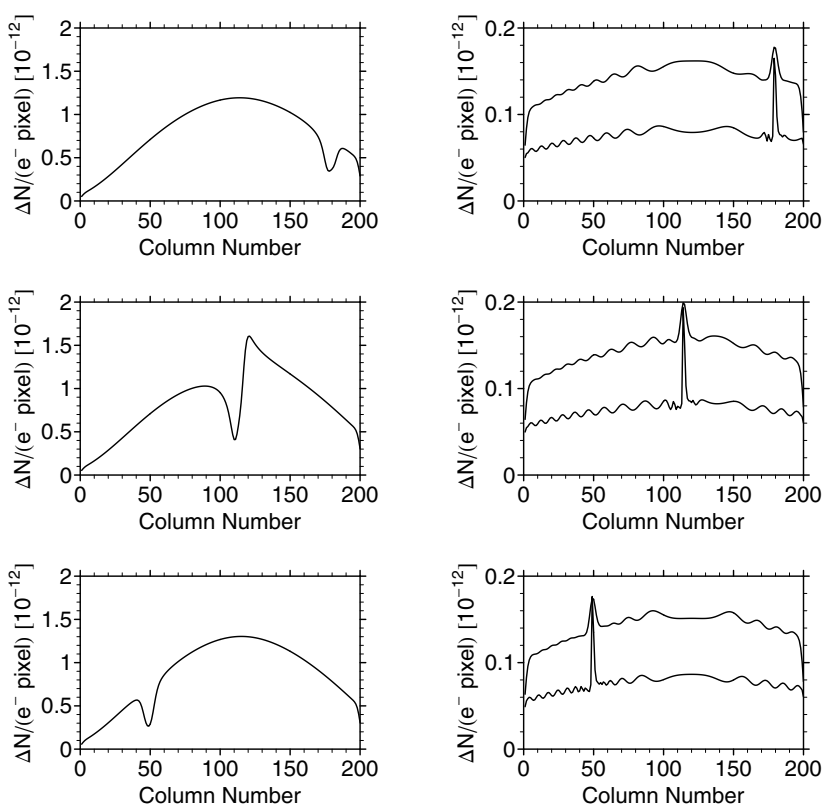

Fig. 16. Results of simulation at photon energy of $10.554 \mathrm{keV}$ and target thickness of $58 \mu \mathrm{m}$ (left) and $1000 \mu \mathrm{m}$ (right). Shown are the number of photons $\Delta N$ per pixel and electron for one row of the pn CCD detector as a function of the column number. From the upper to the lower panel the rotation angle of the target crystal $\psi_{x}$ was varied in steps of $\Delta \psi_{x}=0.5894 \mathrm{mrad}$. The beam spot sizes of the experiment and scattering of the electron beam were taken into account. The residual interference oscillations originate from the interference of the remaining $4 \%$ amplitude created at the entrance interface with the amplitude at the exit interface of the crystal. However, these oscillations are smoothed out in a real experiment in which summation is made over several or all rows of the pn CCD detector.

observation angle $\theta_{x}$. Since its reflecting power of the crystal monochromator exhibits energetically a narrow-band characteristics, quasi-monochromatic intensity structures emitted from the target crystal can be detected by this experimental arrangement.

Experiments were performed with target crystals of varying thickness and for different photon energies. As an example the intensity distributions of the experiment with $58 \mu \mathrm{m}$ and $1 \mathrm{~mm}$ crystal thicknesses are shown in fig. 15 . The most striking features are the structures which move across the pn CCD detector if the rotation angle $\psi_{x}$ of the target crystal around the vertical $y$-axis is varied. These structures are for the thin targets interferences of the radiation amplitudes created at the entrance and exit interfaces of the crystal which originate from a resonance in the dispersion surface of the electron in the crystal. The pronounced peak structures observed for the thick target which are clearly correlated to the interference structures of the thin targets are interpreted as FDPXR contributions to the smooth transition radiation background from the downstream interface of the target crystal.

The interference structures can quantitatively be explained in the framework of the well-known TR production 
mechanism utilizing a generalized formation length for crystalline matter [66]. Within this model, which is based on the formalism described in ref. [67], the resonance is connected to forward-diffracted PXR (FDPXR). Calculated emission spectra of this model, including multiple scattering of the electrons, are displayed in fig. 16. The simulations are in good agreement with the experimental observation.

\section{Conclusion and outlook}

A novel interferometer for soft X-rays has been developed. Intensity oscillations with a high degree of coherence have been observed, not only at the $K$ absorption edge of carbon as described in ref. [18] but with the third harmonics of the undulator radiation also at the $L$ absorption edge of $\mathrm{Ni}$ around $865 \mathrm{eV}$. The polarized undulator radiation can be used to investigate the magneto-optical properties of 3d transition metals. First experiments to determine the $\mathrm{X}$-ray magnetic circular circular dichroism look promising. The energy band between $50 \mathrm{eV}$ and about $1500 \mathrm{eV}$, which can be covered with the interferometer at MAMI, allows the investigation of many elements throughout the periodic table with samples of masses as low as $10 \mathrm{ng}$.

A novel X-ray interferometer has been developed for hard X-rays. The good agreement within experimental errors of our measurements with that of Bonse et al. $[39,40]$ proves that it is fully operational. A simultaneous precision measurement of both, $\delta_{1}(\omega)$ and $\beta_{1}(\omega)$ of the sample foil, should be possible if the foil thicknesses are optimized properly. A possible application of this type of interferometer originates from the transient state of matter which can be produced by multi $\mathrm{GeV}$ bunches of about $1 \mathrm{nC}$ charge and a duration in the $400 \mathrm{fs}$ range. Quite unusual properties are connected with such bunches if focused to a radius $\varrho$ of a few $\mu \mathrm{m}$, e.g. [68]. A strong static electric field of $E_{0} \cong 16 /(\varrho \mu \mathrm{m}) \mathrm{V} / \AA$ is present at the periphery of the charge distribution. In addition, the high electrical current of $2500 \mathrm{~A}$ produces a strong magnetic field $B \cong$ $540 /(\varrho \mu \mathrm{m})$ T. During the passage of such a bunch through a thin foil the matter is put in a transient state which is characterized by a high dielectric polarization in the presence of a strong magnetic field at probably a rather large non-equilibrium electron temperature. This state can, in principle, be investigated by our novel interferometer.

PXR emission from silicon single crystal slabs has been investigated with the electron beam of MAMI. The results of the line width measurements in backward geometry shows that the PXR line widths in Si crystals are superior to the Darwin-Prins widths only for the (111) and (333) reflections. Line broadening effects due to multiple scattering spoil the predicted outstanding resolution for higher-order reflections. With crystals from low- $Z$ materials, like diamond or $\mathrm{LiH}$, the small-angle scattering is reduced and much narrower lines may be expected as for silicon single crystals.

The radiation from silicon single crystals, emitted close to the electron beam direction, has been studied to elucidate the discussion of kinematical versus dynamical pro- duction of PXR. For thin crystals pronounced interference structures in forward direction have been observed when about a Bragg condition was fulfilled. This interference is corroborated by the narrow FDPXR lines observed with a thick Si crystal for which essentially only the exit interface of the target crystal contributes to the observed intensity. Our experimental results show that PXR production is a dynamical rather than a kinematical process.

We thank A. Steinhof for substantial contributions in the redesign and reconstruction of the undulator interferometer and N. Clawiter, S. Dambach, Th. Doerk, M. El-Ghazaly, F. Hagenbuck, G. Kube, A. Rueda and D. Schroff for their help during the course of the experiments described in this contribution. This work has been supported by Deutsche Forschungsgemeinschaft DFG under contract BA 1336/1-4.

\section{References}

1. H. Backe, K.H. Brenzinger, F. Buskirk, S. Dambach, Th. Doerk, N. Eftekhari, H. Euteneuer, F. Görgen, C. Herberg, F. Hagenbuck, K. Johann, K.H. Kaiser, O. Kettig, G. Knies, G. Kube, W. Lauth, B. Limburg, J. Lind, H. Schöpe, G. Stephan, Th. Walcher, Th. Tonn, R. Zahn, in R.L. Johnson, H. Schmidt-Böcking, B.F. Sonntag (Editors), X-Ray and Inner-Shell Processes: 17th International Conference, AIP Conf. Proc. 389 (AIP Press, Woodbury, New York, 1997).

2. F. Hagenbuck, H. Backe, N. Clawiter, H. Euteneuer, F. Görgen, P. Holl, K. Johann, K.-H. Kaiser, J. Kemmer, Th. Kerschner, O. Kettig, H. Koch, G. Kube, W. Lauth, H. Matthäy, M. Schütrumpf, R. Stötter, L. Strüder, Th. Walcher, A. Wilms, C.v. Zanthier, M. Zemter, IEEE Trans. Nucl. Sci. 48, 843 (2001).

3. M. El-Ghazaly, H. Backe, W. Lauth, G. Kube, P. Kunz, A. Sharafutdinov, T. Weber, these proceedings.

4. G. Kube, H. Backe, H. Euteneuer, A. Grendel, F. Hagenbuck, H. Hartmann, K.H. Kaiser, W. Lauth, H. Schöpe, G. Wagner, Th. Walcher, M. Kretzschmar, Phys. Rev. E 65, 056501 (2002).

5. H. Backe, W. Lauth, H. Mannweiler, H. Rochholz, K. Aulenbacher, R. Barday, H. Euteneuer, K.-H. Kaiser, G. Kube, F. Schwellnus, V. Tioukine, in Proceedings of NATO Advanced Radiation Sources and Applications, Nor-Hamberd, Yerevan, Armenia, 2004, edited by H. Wiedemann, NATO Sci. Ser. II, Vol. 199 (Springer, Dortrecht, 2006) p. 267.

6. H. Backe, W. Lauth, A. Rueda, M. El-Ghazaly, P. Kunz, A. Picard, A. Scharafutdinov, A. Sossalla, T. Weber in Topics in Heavy Ion Physics - Proceedings of the Memorial Symp. for G. Soff, Frankfurt, Germany, 2005, edited by W. Greiner, J. Reinhardt, (EP Systema Bt., Budapest, 2005) p. 1

7. A.V. Korol, A.V. Solov'yov, W. Greiner, Int. Jour. Mod. Phys. E-Nucl. Phys. 13, 867 (2004).

8. G. Materik, C.J. Sparks, K. Fischer (Editors), Resonant Anomalous X-ray Scattering (North Holland, Amsterdam, London, New York, Tokyo, 1994).

9. J. Stöhr, NEXAFS Spectroscopy, Springer Ser. Surface Sci., Vol. 25 (Springer, New York, 1992). 
10. B. Lengeler, in [8], p. 35.

11. U. Bonse, M. Hart, Appl. Phys. Lett. 7, 238 (1965).

12. W.K. Warburton, K.F. Ludwig, Phys. Rev. B 33, 8424 (1986).

13. M. Deutsch, M. Hart, Phys. Rev. B 30, 643 (1984).

14. A. Freund, in Anomomalous Scattering, edited by $\mathrm{R}$. Ramaseshan, S.C. Abrahams (Munksgaard Copenhagen, $1975)$ p. 69.

15. N. Kato, S. Tanemura, Phys. Rev. Lett. 19, 22 (1967).

16. R.L Blake, J.C. Davis, D.E. Graessle, T.H. Burbine, E.M. Gullikson, in [8], p. 79.

17. F. Polack, D. Joyeux, J. Svatos, D. Phalippou, Rev. Sci. Instrum. 66, 2180 (1995).

18. S. Dambach, H. Backe, Th. Doerk, N. Eftekhari, H. Euteneuer, F. Görgen, F. Hagenbuck, K.H. Kaiser, O. Kettig, G. Kube, W. Lauth, H. Schöpe, A. Steinhof, Th. Tonn, Th. Walcher, Phys. Rev. Lett. 80, 5473 (1998).

19. M. Itou, T. Harada, T. Kita, Appl. Optics 28, 146 (1989).

20. R. Soufli, E.M. Gullikson, Appl. Opt. 36, 5499 (1997).

21. N. Kerr Del Grande, Physica Scripta 41, 110 (1990).

22. J. Stöhr, J. Magn. Magn. Mater. 200, 470 (1999).

23. G. Schütz, W. Wagner, W. Wilhelm, P. Kienle, R. Zeller, R. Frahm, G. Materlik, Phys. Rev. Lett. 58, 737 (1987).

24. J.B. Kortright, Sang-Koog Kim, Phys. Rev. B 62, 12216 (2000).

25. http://www . andor-tech. com/germany/products/oem. cfm.

26. H. Soltau, P. Holl, J. Kemmer, S. Krisch, C.v. Zanthier, D. Hauff, R. Richter, H. Bräuninger, R. Hartmann, G. Hartner, N. Krause, N. Meidinger, E. Pfeffermann, C. Reppin, G. Schwaab, L. Strüder, J. Trümper, E. Kendziorra, J. Krämer, Nucl. Instrum. Methods A 377, 340 (1996).

27. M.L. Cherry, G. Hartmann, D. Müller, T.A. Prince, Phys. Rev. D 10, 3594 (1974).

28. M.J. Moran, B.A. Dahling, P.J. Ebert, M.A. Piestrup, B.L. Bergman, J.O. Kephart, Phys. Rev. Lett. 57, 1223 (1986).

29. P. Goedtkind, J.-M. Salomé, X. Atru, P. Dhez, M. Jablonka, N. Maene, F. Poortmans, L. Wartski, Nucl. Instrum. Methods B 56/57, 1060 (1991).

30. M.A. Piestrup, D.G. Boyers, C.I. Pincus, Qiang Li, G.D. Hallewell, M.J. Moran, D.M. Skopik, R.M. Silzer, X.K. Maruyama, D.D. Snyder, G.B. Rothbart, Phys. Rev. A 45, 1183 (1992).

31. V.V. Kaplin, V.N. Zabaev, E.I. Rozum, S.R. Uglov, S.A. Vorobiev, Phys. Lett. A 174, 165 (1993).

32. H. Backe, S. Gampert, A. Grendel, H.J. Hartmann, W. Lauth, Ch. Weinheimer, R. Zahn, F.R. Buskirk, H. Euteneuer, K.H. Kaiser, G. Stephan, Th. Walcher, Z. Phys. A 349, 87 (1994).

33. O. Kettig, H. Backe, N. Clawiter, S. Dambach, Th. Doerk, N. Elbai, H. Euteneuer, F. Hagenbuck, P. Holl, H. Jacobs, K.H. Kaiser, J. Kemmer, Th. Kerschner, G. Kube, H. Koch, W. Lauth, H. Mannweiler, H. Matthäy, H. Schöpe, D. Schroff, M. Schüttrumpf, R. Stötter, L. Strüder, Th. Walcher, A. Wilms, C.v. Zanthier, M. Zemter, in R.W. Dunford, D.S. Gemmell, E.P. Kanter, B. Krässig, S.H. Southworth, L. Young (Editors) X-ray and Inner-Shell Processes: 18th International Conference, AIP Conf. Proc. 506 (AIP Press, Melville, New York, 2000).

34. O. Kettig, Dissertation, Institut für Kernphysik, Universität Mainz, 2000 (in German).

35. H. Backe, N. Clawiter, S. Dambach, H. Euteneuer, F. Hagenbuck, K.-H. Kaiser, O. Kettig, G. Kube, W. Lauth, Th.
Walcher, in Proceedings of the International Conference on Fundamenttal and Applied Aspects of Modern Physics, Lüderitz, Namibia, 2000, edited by S.H. Connell, R. Tegen (World Scientific, New Jersey, London, Singapore, Hong Kong, 2001) p. 123.

36. O. Kettig et al., to be published.

37. B.L. Henke, E.M. Gullikson, J.C. Davis, Atom. Data and Nucl. Data Tabl. 54, 181 (1993).

38. E. Storm, H.I. Israel, Atom. Data and Nucl. Data Tabl. A 7, 565 (1970).

39. U. Bonse, G. Materlik, Z. Phys. B 24, 189 (1976).

40. U. Bonse, I. Hartmann-Lotsch, Nucl. Instrum. Methods 222, 185 (1984).

41. V.G. Baryshevsky, Dokl. Akad. Nauk BSSR 15, 306 (1971).

42. V.G. Baryshevsky, I.D. Feranchuk, Zh. Exper. Teor. Fiz. 61, 944 (1971); (Sov. Phys. JETP 34, 50 (1972); addendum, ibid 64, 760 (1973)).

43. G.M. Garibian, C. Yang, Zh. Eksp. Teor. Fiz. 61, 930 (1971), (Sov. Phys. JETP 34, 495 (1972)).

44. G.M. Garibian, C. Yang, Zh. Eksp. Teor. Fiz. 63, 1198 (1972), (Sov. Phys. JETP 36, 631 (1973)).

45. M. Ter-Mikaelian, High-Energy Electromagnetic Processes in condensed Media (Wiley-Interscience, New York, London, Sydney, Toronto, 1972).

46. K.-H. Brenzinger, C. Herberg, B. Limburg, H. Backe, S. Dambach, H. Euteneuer, F. Hagenbuck, H. Hartmann, K. Johann, K.H. Kaiser, O. Kettig, G. Knies, G. Kube, W. Lauth, H. Schöpe, Th. Walcher, Z. Phys. A 358, 107 (1997).

47. J. Freudenberger, H. Genz, V.V. Morokhovskyi, A. Richter, J.P.F. Sellschop, Phys. Rev. Lett. 84, 270 (2000).

48. H. Backe, G. Kube, W. Lauth, Proceedings of NATO Advanced Research Workshop on Electron-Photon Interaction in Dense Media, Nor-Hamberd, Yerevan, Armenia, 2001, edited by H. Wiedemann, NATO Sci. Ser. II, Vol. 49 (Kluwer Academic Publishers, Dordrecht, Boston, London, 2001) p. 153.

49. 5th International Symposium on Radiation from Relativistic Electrons in Periodic Structures (RREPS-01), Lake Aya, Russia, September, 2001, Topical Issue of Nucl. Instrum. Methods Phys. Res. B 201, 1 (2003).

50. X. Artru, P. Rullhusen, Nucl. Instrum. Methods Phys. Res. B 145, 1 (1998); addendum, ibid 173, 16 (2001).

51. V. Baryshevsky, I. Feranchuk, J. Phys. (Paris) 44, 913 (1983).

52. I. Feranchuk, A. Ivashin, J. Phys. (Paris) 46, 1981 (1985).

53. H. Nitta, Phys. Lett. A 158, 270 (1991).

54. A. Caticha, Phys. Rev. B 45, 9541 (1992).

55. A.V. Shchagin, V.I. Pristupa, N.A. Khizhnyak, Phys. Lett. A 148, 485 (1990).

56. J. Freudenberger, V.B. Gavrikov, M. Galemann, H. Genz, L. Groening, V.L. Morokhovskii, V.V. Morokhovskii, U. Nething, A. Richter, J.P.F. Sellschop, N.F. Shulga, Phys. Rev. Lett. 74, 2487 (1995).

57. O.M. Lugoskaya, S.A. Stepanov, Sov. Phys. Crystallogr. 36, 478 (1991) and http://sergey.gmca.aps.anl.gov/ cgi/XOh.html.

58. J. Freudenberger, H. Genz, V.V. Morokhovskii, A. Richter, V.L. Morokhovskii, U. Nething, R. Zahn, J.P.F. Sellschop, Appl. Phys. Lett. 70, 267 (1997).

59. K.-H. Brenzinger, B. Limburg, H. Backe, S. Dambach, H. Euteneuer, F. Hagenbuck, C. Herberg, K.H. Kaiser, 
O. Kettig, G. Kube, W. Lauth, H. Schöpe, Th. Walcher, Phys. Rev. Lett. 79, 2462 (1997).

60. H. Backe, C. Ay, N. Clawiter, Th. Doerk, M. El-Ghazaly, K.-H. Kayser, O. Kettig, G. Kube, F. Hagenbuck, W. Lauth, A. Rueda, A. Scharafutdinov, D. Schroff, T. Weber in: W. Greiner, A. Solov'yov, S. Misicu (Editors) Proceedings Symp. Channeling - Bent Crystals - Radiation Processes, Frankfurt (Germany) 2003 (EP Systema, Debrecen, 2003) p. 41.

61. H. Nitta, J. Phys. Soc. Jpn. 69, 3462 (2000).

62. V.G. Baryshevsky, Nucl. Instrum. Methods Phys. Res. B 122, 13 (1997).

63. A. Kubankin, N. Nasonov, V. Sergienko, I. Vnukov, Nucl. Instrum. Methods Phys. Res. B 201, 97 (2003).
64. N. Nasonov, V. Sergienko, N. Noskov, Nucl. Instrum. Methods Phys. Res. B 201, 67 (2003).

65. N. Aleinik, A.N. Baldin, E.A. Bogomazova, I.E. Vnukov, B.N. Kalinin, A.S. Kubankin, N.N. Nasonov, G.A. Naumenko, A.P. Potylitsyn, A.F. Sharafutdinov, JETP Lett. 80, 393 (2004) (Pis'ma Zh. Eksp. Teor. Fiz. 80, 447 (2004))

66. H. Backe, A. Rueda, W. Lauth, N. Clawiter, M. ElGhazaly, P. Kunz, T. Weber, Nucl. Instrum. Methods Phys. Res. B 234, 138 (2005).

67. A. Caticha, Phys. Rev. A 40, 4322 (1989).

68. C.D. Back, D. Weller, J. Heidmann, D. Mauri, D. Guarisco, E.L. Garwin, H.C. Siegmann, Phys. Rev. Lett. 81, 3251 (1998). 